\title{
Adaptação brasileira da Coping with Children's Negative Emotions Scale (CCNES)
}

\author{
Taiane Lins ${ }^{1}$, Patrícia Alvarenga \\ Universidade Federal da Bahia, Salvador-BA, Brasil \\ Deise Maria Leal Fernandes Mendes \\ Universidade Estadual do Rio de Janeiro, Rio de Janeiro-RJ, Brasil \\ Luciana Fontes Pessôa \\ Pontifícia Universidade Católica do Rio de Janeiro, Rio de Janeiro-RJ, Brasil
}

\section{RESUMO}

O objetivo deste estudo foi adaptar a Coping with Children's Negative Emotions Scale (CCNES) para a população brasileira e verificar seus parâmetros psicométricos preliminares. O estudo foi desenvolvido em seis etapas: tradução da escala original; avaliação da escala traduzida por especialistas; avaliação da escala traduzida pelo público-alvo em grupos focais; retrotradução; piloto; coleta de dados com cem mães de crianças entre três e oito anos; avaliação da consistência interna e das correlações entre as sete reações maternas investigadas. Foram obtidos valores satisfatórios de consistência interna, com exceção das reações de desconforto. Além disso, as dimensões de reações maternas apresentaram correlações significativas em cada uma das subescalas (práticas apoiadoras e não apoiadoras da expressão emocional infantil). A avaliação preliminar da CCNES indica seu bom funcionamento, sinalizando que a versão adaptada pode ser submetida ao processo de validação.

Palavras-chave: adaptação; CCNES; socialização emocional.

\section{ABSTRACT - Brazilian Adaptation of the Coping with Children's Negative Emotions Scale (CCNES)}

The objective of this study was to adapt the Coping with Children's Negative Emotions Scale (CCNES) to the Brazilian population and check its preliminary psychometric parameters. The study was developed in six stages: translation of the original scale; assessment of the scale translated by experts; assessment of the scale translated by the target audience in focus groups; back-translation; pilot; data collection with one hundred mothers of children aged between three and eight years; evaluation of internal consistency and correlations among the seven investigated maternal reactions. Satisfactory internal consistency values were obtained, except for the discomfort reactions. Furthermore, the dimensions of maternal reactions showed significant correlations in each of the subscales (supportive and non-supportive practices of children's emotional expression). The preliminary assessment of CCNES indicates its good operation, signaling that the adapted version can be submitted to the validation process.

Keywords: adaptation; CCNES; emotion socialization.

RESUMEN - Adaptación brasileña de la Coping with Children's Negative Emotions Scale (CCNES)

El objetivo del estudio es presentar la versión traducida al portugués y adaptada de la Coping with Children's Negative Emotions Scale (CCNES) y comprobar sus parámetros psicométricos preliminares. El estudio se desarrolló en seis etapas: traducción de la escala original; evaluación de la escala traducida por expertos; evaluación de la escala traducida en grupos focales; retrotraducción; piloto; recopilación de datos con cien madres de niños de entre tres y ocho años; evaluación de la consistencia interna y de las correlaciones entre las siete reacciones maternas investigadas. Se obtuvieron resultados satisfactorios de consistencia interna, con excepción de las reacciones de molestias. Además, las dimensiones de las reacciones maternas mostraron correlaciones significativas en cada una de las subescalas (la práctica de apoyo y de no apoyo a la expresión emocional del niño). La evaluación preliminar de CCNES indica su buen funcionamiento, señalando que la versión adaptada puede ser sometida al proceso de validación.

Palabras clave: adaptación; CCNES; socialización emocional.

As reações parentais às emoções manifestadas pela criança, em especial aquelas consideradas em seu contexto social como negativas (raiva, medo e tristeza, por exemplo), têm sido vistas como importantes estratégias de socialização emocional (Eisenberg, Cumberland, \&
Spinrad, 1998). Tais emoções, uma vez encaradas como inadequadas ou indesejáveis em seu meio social, configuram um desafio para a criança que precisa aprender a lidar com elas (Ramsden \& Hubbard, 2002). Assim, a partir dos anos pré-escolares, ou segunda infância, a 
forma como os pais lidam com as próprias emoções e com as dos filhos parece se constituir como uma fonte de aprendizagem e experiência particularmente relevante (Sroufe, 1996). Nessa etapa do desenvolvimento, a criança está aprendendo e começando a apresentar habilidades para se autorregular emocionalmente, que se fazem cada vez mais necessárias, pois está se envolvendo em interações sociais gradativamente mais complexas, e ampliando seu raio de atuação em diferentes contextos, criando a necessidade de aprender a lidar com emoções diversas, suas e dos pares, em situações variadas (Havighurst, Wilson, Harley, \& Prior, 2009; Morris, Silk, Steinberg, Myers, \& Robinson, 2007).

A expansão desse campo de estudos veio acompanhada da necessidade de avanços metodológicos, incluindo a busca por instrumentos para avaliação de aspectos da socialização emocional envolvendo a emocionalidade parental e a reação dos pais. Algumas das alternativas que devem ser mencionadas pelo seu emprego em estudos com esse foco de interesse são o Maternal Emotional Styles Questionnaire (MESQ) (Lagacé-Séguin \& Coplan, 2005), o Self-Expressiveness in the Family Questionnaire (SEFQ) (Halberstadt, Parke, Cassidy, Stifter, \& Foz, 1995) e a Coping with Children's Negative Emotions Scale (CCNES) (Fabes, Eisenberg, \& Bernzweig, 1990). A CCNES merece destaque, especialmente por estar sendo utilizada em estudos realizados com populações de diferentes países como Turquia, Índia, China, além dos Estados Unidos e de países da Europa, obtendo bons indicadores de consistência interna (Altan-Aytun, Yagmurlu, \& Yavuz, 2013; Alves \& Cruz, 2011; Cassano, Zeman, \& Sanders, 2014; Raval \& Martini, 2011; Tao, Zhou, \& Wang, 2010).

A versão original da escala proposta por Fabes et al. (1990) é constituída por 12 situações hipotéticas que incluem reações emocionais da criança consideradas negativas. Aos pais é solicitado que procurem imaginar-se nas situações e, em seguida, respondam a itens que se referem a cada uma das seis reações ou práticas a serem por eles avaliadas, que podem ser agrupadas como práticas que apoiam e que não apoiam a experiência emocional da criança. Entre as que não apoiam as emoções da criança estão: reações de desconforto, reações punitivas ou recriminatórias e reações que minimizam as emoções negativas dos filhos. Por outro lado, as práticas avaliadas que apoiam as emoções são: reações centradas nas emoções, reações centradas no problema e reações de incentivo. As propriedades psicométricas dessa escala foram avaliadas por Fabes, Poulin, Eisenberg e Madden-Derdich (2002) em estudo realizado com população residente nos Estados Unidos, tendo como participantes 96 mães e cinco pais de crianças entre três e seis anos. De acordo com os achados, os valores de alfa de Cronbach para as dimensões são considerados satisfatórios. Os valores de alfa de Cronbach encontrados para cada reação parental foram: 0,70 para reações de desconforto; 0,69 para reações punitivas; 0,85 para reações de incentivo; 0,80 para reações centradas nas emoções; 0,78 para reações centradas no problema; e 0,78 para reações que minimizam as respostas emocionais da criança.

Adaptações da escala foram realizadas em alguns países, o que parece sinalizar o reconhecimento da relevância do uso desse instrumento. Coutu, Dubeau, Provost, Royer e Lavigueur (2002), por exemplo, adaptaram e validaram uma versão francesa e verificaram que todas as reações parentais investigadas no instrumento obtiveram valores de alfa acima de 0,70 , com exceção das reações de desconforto, que obtiveram alfas de Cronbach de 0,50 , de acordo com a participação das mães, e de 0,59 , de acordo com a participação dos pais. Participaram do estudo de Coutu et al. (2002) 220 mães e 154 pais de crianças com idade média de 4,5 anos. Melo (2005), em estudo que envolveu a participação de 134 mães e 123 pais portugueses de crianças entre sete e nove anos para a adaptação de uma versão reduzida da CCNES, com apenas oito situações, verificou valores de alfa de Cronbach acima de 0,73 para reações que minimizam, reações de incentivo, reações centradas nas emoções e reações centradas no problema. Contudo, para as reações de desconforto e as reações punitivas, os valores de alfa foram respectivamente de 0,42 e 0,59. Altan-Aytun et al. (2013) adaptaram essa escala para avaliar as práticas de socialização emocional adotadas por mães turcas. Mães de 141 crianças entre quatro e seis anos participaram do estudo, o qual encontrou valores de alfa de Cronbach acima de 0,72 para todas as reações maternas, com exceção das reações de desconforto, que obtiveram alfa de 0,65 . De forma geral, verifica-se que as diferentes adaptações da CCNES têm obtido valores de alfa de Cronbach considerados aceitáveis.

Mirabile (2015) avaliou a inclusão na escala original da dimensão reações que ignoram as emoções da criança, em referência a situações em que os pais não respondem às manifestações emocionais dos filhos ou não sabem lidar com elas. De acordo com os achados, verificou-se correlação positiva entre essa dimensão e as dimensões da CCNES que se referem às reações que não apoiam a expressão de emoções negativas da criança (reações de desconforto, punitivas e que minimizam as respostas emocionais).

Considerando a relevância do estudo de construtos ligados às cognições e práticas parentais relacionadas às emoções e a carência de instrumentos para esse tipo de investigação no Brasil, o objetivo deste estudo foi adaptar a CCNES para a população brasileira e verificar seus parâmetros psicométricos preliminares. Em virtude do grande esforço metodológico requerido para um estudo de validação, é recomendável que a adaptação cultural e linguística de instrumentos criados em outros países seja realizada de forma criteriosa e que as características do produto gerado sejam cuidadosamente avaliadas antes de proceder análises mais sofisticadas com grande número de participantes. Por essas razões, o presente 
estudo apresenta o processo de adaptação cultural e linguística da escala e analisa sua consistência interna e as correlações entre as dimensões para que, em um segundo estudo, valide-se a versão adaptada do instrumento. A hipótese foi de que as dimensões de reações maternas teriam índices de consistência interna acima de 0,70 . Além disso, esperava-se que existiriam correlações positivas entre as diferentes dimensões de práticas apoiadoras da experiência emocional da criança e entre as dimensões de práticas não apoiadoras da experiência emocional da criança.

\section{Método}

\section{Coping with Children's Negative Emotions Scale}

A CCNES (Fabes et al., 1990) é uma escala que apresenta 12 situações hipotéticas envolvendo eventos comuns à infância e que tendem a contribuir para a vivência de emoções consideradas negativas, como raiva, medo, ansiedade, tristeza, frustração, entre outras. Como exemplos dessas situações estão: a perda do brinquedo favorito, tomar injeção, ficar longe dos pais, fazer apresentações em público e ser rejeitado por pares. Um exemplo completo de situação que constitui a escala é: "Se meu filho fica irritado porque está doente ou machucado e não pode ir à festa de aniversário de seu amigo, eu...”. Cada situação é seguida por seis afirmativas que devem ser respondidas com base em uma escala do tipo Likert de sete pontos, que varia de muito improvável a muito provável. O agrupamento dos diferentes tipos de reações parentais configura duas subescalas. A subescala de práticas que não apoiam as emoções da criança é formada pelas seguintes dimensões:

- reações de desconforto (referem-se às respostas caracterizadas pelo incômodo dos pais diante das emoções negativas expressas pelo filho);

- reações punitivas ou recriminatórias (indicam punições ou recriminações utilizadas pelos pais com o objetivo de reduzir ou mesmo inibir a expressão emocional da criança);

- reações que minimizam as respostas emocionais (indicam o quanto os pais buscam reduzir a importância ou a seriedade da situação ou da emoção expressa pelo filho).

Por outro lado, a subescala de práticas que apoiam as emoções negativas dos filhos é composta pelas seguintes dimensões:

- reações centradas nas emoções (indicam estratégias parentais que têm o propósito de auxiliar o filho a lidar com as emoções);

- reações centradas no problema (referem-se às estratégias parentais que ajudam o filho a resolver as situações que levaram à manifestação das emoções negativas);

- reações de incentivo (buscam estimular e validar as expressões emocionais dos filhos).
Destaca-se ainda que a versão utilizada para a adaptação contempla a dimensão reações que ignoram as emoções da criança, adicionada à versão original de Fabes et al. (1990) e avaliada por Mirabile (2015). Essa escala pode tornar o instrumento mais completo, abrangendo uma diversidade maior de possibilidades de reações parentais frente às emoções negativas dos filhos. A escala pode ser autoadministrada, e o escore de cada tipo de reação e de cada subescala é obtido por meio da média das pontuações nas respostas dadas pelos respondentes a cada item nas 12 situações. A escala não fornece, contudo, escore total ou geral de práticas de socialização emocional. Salienta-se ainda que os itens que avaliam as reações são positivos, com exceção de quatro itens que compõem a dimensão reações de desconforto, os quais, por serem negativos, são avaliados de forma inversa (exemplo: "Mantenho a calma e não me deixo ficar triste como ele").

\section{Primeira fase}

Adaptação linguística e cultural da Coping with Children's Negative Emotions Scale. A adaptação da CCNES foi iniciada a partir da autorização do primeiro autor do instrumento, e o processo de adaptação linguística e cultural foi baseado nos critérios propostos por Borsa, Damásio e Bandeira (2012), Manzi-Oliveira, Balarini, Marques, e Pasian (2011), Reis, Oliveira, Bandeira, e Sperb (2013) para adaptação de instrumentos de avaliação. O processo de adaptação linguística e cultural da CCNES foi realizado em cinco etapas: tradução da escala original; síntese das versões traduzidas e avaliação por especialistas; avaliação da escala traduzida em grupos focais; retrotradução; e avaliação piloto. Essas etapas são descritas a seguir:

Tradução da escala e síntese das versóes traduzidas: A primeira etapa envolveu a tradução da escala para o português por um tradutor bilíngue, nativo em língua inglesa, que desconhecia a escala e o vocabulário da área investigada; e por um tradutor com conhecimento técnico na área. Em seguida, dois especialistas no tema fizeram uma síntese das duas versões traduzidas, buscando reunir as melhores opções de tradução, considerando a coerência e a clareza do texto, assim como o uso de linguagem mais simples que tornasse o entendimento da escala mais fácil por diferentes públicos.

Avaliação da escala por especialistas: Posteriormente, a síntese foi avaliada por oito especialistas no tema e em avaliação psicológica. Os especialistas responderam a um questionário estruturado desenvolvido pelas autoras do estudo. De acordo com esse questionário, os especialistas deveriam avaliar a adequação dos seguintes aspectos: número de situações apresentadas, número de afirmativas, amplitude da escala Likert utilizada, diagramação da escala, instrução oferecida aos participantes sobre como responder à escala e adequação da linguagem para pessoas de diferentes níveis socioculturais. Além disso, como na 
elaboração da síntese surgiram dúvidas sobre a adequação da tradução de três expressões ("not to make a big deal" para "não fazer um drama"; "is over-reacting" para "está exagerando"; "it's ok to cry" para "tudo bem chorar"), os especialistas deveriam responder também se a expressão em português poderia ser compreendida pela maioria das pessoas em nossa cultura.

Avaliação da escala em grupos focais: Após as revisões feitas em decorrência das contribuições dos especialistas, a versão resultante da CCNES foi avaliada por mães e pais em dois grupos focais, um realizado na cidade do Rio de Janeiro e outro em Salvador, Bahia, cada um conduzido por duas moderadoras. No primeiro grupo, participaram dois pais e duas mães de crianças entre seis e oito anos e, no segundo, duas mães e um pai de crianças entre três e cinco anos. Nos grupos, os pais foram solicitados a apontar dúvidas, palavras ou termos ambíguos e inadequados, assim como a avaliar a diagramação da escala. Os participantes dos dois grupos possuíam características socioculturais distintas, de forma a contemplar maior variedade de respondentes. Todas as sugestões apresentadas pelos participantes foram discutidas por todo o grupo e registradas por uma das moderadoras para alteração posterior na escala, considerando o consenso atingido pelo grupo após a discussão das dúvidas e impressões sobre os itens.

Retrotradução: A versão da CCNES em língua portuguesa foi traduzida para a língua inglesa por um tradutor bilíngue sem conhecimento técnico do tema avaliado. A partir da versão retrotraduzida, foi feita uma avaliação de compatibilidade entre a versão adaptada e a versão em língua inglesa do instrumento. Essa avaliação foi realizada pelas pesquisadoras do presente estudo, que calcularam o percentual de palavras da versão produzida no processo de retrotradução que eram iguais às palavras da versão original da escala.

Avaliação piloto: Por fim, a última etapa do processo de adaptação linguística e cultural da CCNES envolveu a realização de um teste piloto da escala para a avaliação preliminar da linguagem e da apresentação do instrumento. Nessa etapa, participaram três pais e três mães de crianças entre seis e oito anos, na cidade do Rio de Janeiro, e três pais e quatro mães de crianças entre três e cinco anos, na cidade de Salvador, Bahia. Esses participantes foram selecionados por acessibilidade e, assim como no grupo focal, possuíam características socioculturais distintas. Os participantes responderam à escala individualmente e, após a finalização, foram questionados pela pesquisadora se tiveram dúvidas e se alguma frase ou expressão não ficou clara.

\section{Segunda fase}

Avaliação preliminar das propriedades
psicométricas da Coping with Children's Negative
Emotions Scale. Participantes: Após os ajustes
finais, foi realizada a coleta de dados apenas com mães
para a avaliação das propriedades psicométricas da

versão adaptada da CCNES. Participaram do estudo 100 mães, com média de idade de 36,2 anos $(D P=7,2)$, sendo 47 residentes na cidade do Rio de Janeiro e 53 na cidade de Salvador. A escolaridade das participantes, avaliada pelo número de anos de estudo, foi, em média, de 13,7 anos $(D P=2,90)$. As crianças (47 meninas e 53 meninos) tinham entre três e oito anos de idade $(M=5,50 ; D P=1,82)$ e residiam, em sua maioria, com os pais e os irmãos (57\%).

Procedimentos de coleta de dados: O projeto foi apresentado a escolas públicas e particulares de ambas as cidades. A partir da autorização das escolas e do Comitê de Ética em Pesquisa (CEP) da Universidade Federal do Estado do Rio de Janeiro (UNIRIO), mães de crianças entre três e cinco anos, residentes em Salvador, e de crianças entre seis e oito anos, residentes no Rio de Janeiro, foram convidadas a participar do estudo. Aquelas que aceitaram assinaram duas vias do Termo de Consentimento Livre e Esclarecido (TCLE), além de responderem a uma Ficha de Dados Sociodemográficos e preencherem a versão adaptada da CCNES.

\section{Instrumentos}

Ficha de dados sociodemográficos: Foi utilizada para a obtenção de informações da família, como escolaridade, profissão, idade dos pais, renda familiar, existência de outros filhos, entre outras.

CCNES: A versão utilizada é resultante do processo de adaptação linguística e cultural da escala original, descrito na seção anterior.

\section{Considerações éticas}

O presente estudo foi submetido e aprovado pelo CEP da Universidade Federal do Estado do Rio de Janeiro (UNIRIO) em maio de 2015 ( $\mathrm{n}^{\mathrm{o}}$ 1.102.424), de acordo com as diretrizes da Resolução no 466/12 do Conselho Nacional de Saúde a respeito das pesquisas envolvendo seres humanos.

\section{Resultados}

\section{Primeira fase}

Adaptação linguística e cultural da Coping with Children's Negative Emotions Scale. Tradução da escala e síntese das versões traduzidas: As versões traduzidas foram avaliadas por dois especialistas, os quais elaboraram uma síntese que constituiu uma terceira versão, com as melhores opções de tradução de cada situação e de suas alternativas de resposta.

Avaliação da escala por especialistas: A versão com a síntese das traduções foi avaliada por oito especialistas. O número total de situações, a formatação e apresentação do instrumento e a tradução das três expressões que levantavam dúvidas quanto à tradução adotada ("not to make a big deal" para "não fazer um drama"; "is over-reacting" para "está exagerando"; "it’s ok to cry" para "tudo 
bem chorar") foram considerados adequados pelos oito juízes. O número de afirmativas (sete) com as possíveis reações parentais foi considerado insuficiente por um dos juízes. A amplitude da escala Likert foi considerada excessiva por dois juízes. A instrução da escala foi considerada confusa e extensa por um juiz. Por fim, um juiz considerou que a linguagem não poderia ser compreendida por pessoas de diferentes níveis socioculturais. A partir dessa avaliação, optou-se por reduzir a escala Likert de sete para cinco pontos. O número de afirmativas com reações parentais foi mantido por contemplar as sete categorias de reações que apoiam e que não apoiam a experiência emocional da criança. A linguagem utilizada na instrução e nas 12 situações foi mantida para avaliação posterior pelos grupos focais.

Avaliação da escala em grupos focais: Várias expressões e palavras utilizadas na descrição das 12 situações e nas afirmativas que continham as possíveis reações parentais foram questionadas pelos pais nos dois grupos focais. Com base nesses questionamentos e apontamentos, optou-se, inicialmente, por alterar a variação da escala Likert de muito improvável a muito provável para nunca a certamente. Além disso, na descrição das 12 situações hipotéticas, a palavra "trêmulo", que aparecia na situação 4, foi substituída por "nervoso", e o trecho "(meu filho) se assusta com estranhos", da situação 12, foi alterado para "(meu filho) estranha pessoas desconhecidas ou que não tem muito contato". Por fim, nas afirmativas que apresentam as possíveis reações parentais, foram realizadas diversas alterações em palavras que os participantes consideraram ambíguas, desconhecidas ou pouco utilizadas, especialmente para pessoas com baixo nível de instrução. Destacam-se as seguintes alterações nessas afirmativas: "digo para meu filho não fazer um drama" para "digo para meu filho que deixe de drama"; "incentivo meu filho a mostrar seus sentimentos" para "digo ao meu filho para mostrar seus sentimentos"; "não me deixo ficar abalado como ele" para "não me deixo ficar triste como ele"; "ignoro meu filho chorando até ele parar" para "deixo meu filho chorando até ele parar"; "digo a meu filho que tudo bem se ele chorar quando estiver triste" para "digo a meu filho: 'pode chorar, filho!"; "me sinto triste e desconfortável" para "me sinto triste e incomodado".

Retrotradução: Duas autoras do artigo avaliaram a compatibilidade entre a versão retraduzida para o inglês e a versão original do instrumento. Inicialmente, o percentual de palavras iguais nas duas versões ficou muito baixo, em torno de $48 \%$. Em função disso, foi realizada uma revisão da escala, considerando as sugestões dos grupos focais e buscando, além da correspondência com os itens originais da escala, a adaptação da linguagem baseada em especificidades culturais. Em alguns itens, a tradução proposta não apresentou, na retrotradução, correspondência perfeita com o termo original, mas foi mantida por representar do melhor modo o significado pretendido. Assim é que a expressão "shape up", por exemplo, foi traduzida para "comportar-se" na versão original e para "behave" na retrotradução. Considerou-se que "shape up" corresponde a "comportar-se", no sentido de "comporte-se bem". As expressões não usuais foram adaptadas e contextualizadas para melhor entendimento dos participantes. Em decorrência dos ajustes realizados, o índice final de correspondência entre a retrotradução e a versão original aumentou para $78 \%$.

Avaliação piloto: Os participantes não relataram dúvidas ou dificuldades para responder ao questionário, mas sinalizaram a necessidade de pequenos ajustes na formatação e erros de digitação, que foram corrigidos e ajustados.

\section{Segunda fase}

\section{Avaliação preliminar das propriedades psicométricas da Coping with Children's Negative Emotions Scale. A consistência interna das subescalas} e das dimensões da CCNES foi avaliada por meio do coeficiente alfa de Cronbach. Foram realizadas, também, análises de correlação entre as reações. Análises mais refinadas, que requerem um quantitativo maior de participantes, estão planejadas para o estudo de validação da escala, que será a próxima etapa do projeto em que se insere o presente estudo.

De acordo com as análises de confiabilidade, o conjunto de práticas que não apoiam a expressão de emoções negativas dos filhos obteve coeficiente alfa de Cronbach de 0,85 , enquanto o conjunto de práticas que apoiam a expressão das emoções negativas pelos filhos apresentou coeficiente de consistência interna de 0,80 .

Por sua vez, as dimensões que compõem a subescala de práticas que não apoiam a expressão de emoções negativas dos filhos - reações punitivas, reações que minimizam, reações que ignoram as respostas emocionais dos filhos e reações de desconforto - obtiveram, respectivamente, os seguintes valores de alfa: 0,73 , 0,77, 0,69 e 0,16. Verifica-se que, de forma geral, os valores de alfa obtidos para as práticas que não apoiam a expressão de emoções negativas pelos filhos podem ser considerados aceitáveis (Field, 2005), com exceção das reações de desconforto, cujo valor obtido foi extremamente baixo $(0,16)$. Como essa dimensão envolve quatro itens negativos, esses foram retirados da análise para avaliação exploratória de seus efeitos sobre a consistência interna. Contudo, apesar da elevação no valor, o resultado encontrado ainda não foi satisfatório $(0,46)$, o que motivou a realização de uma análise de correlação entre os itens e o escore geral da dimensão, como indicado por Tavakov e Dennick (2011). Verificou-se que todas as relações foram positivas e significativas, o que pode ser um bom indicativo para a dimensão, apesar do baixo valor do alfa. A Tabela 1 apresenta os detalhes dessas correlações. 
Os coeficientes das dimensões que correspondem às reações apoiadoras da expressão emocional dos filhos foram de 0,74 para as reações centradas nas emoções e para as reações que incentivam a expressão de emoções e de 0,60 para as reações centradas no problema. De acordo com Field (2005), desses valores, apenas o coeficiente da dimensão reações centradas no problema seria considerado pouco aceitável.

Visto que não seria possível realizar uma análise de componentes principais com os dados preliminares, foi realizada uma análise de correlação entre as dimensões para continuar explorando a consistência interna da escala. Verificou-se que, entre as práticas que não apoiam a expressão de emoções negativas pelos filhos, as quatro dimensões (reações punitivas, reações que ignoram, reações que minimizam e reações de desconforto) se correlacionam de forma positiva. Contudo, apenas entre as reações punitivas e as reações que minimizam as emoções $(r=0,58 ; p<0,01)$ e entre as reações punitivas e as reações de desconforto $(r=0,57 ; p<0,01)$ as correlações foram moderadas. Já as outras correlações foram de fraca intensidade, variando de 0,23 a $0,39(p<0,01)$. Entre as práticas que apoiam a expressão de emoções negativas pelos filhos, foram verificadas correlação positiva e de intensidade moderada entre as reações centradas no problema e as reações centradas nas emoções $(r=0,50 ; p<0,01)$ e correlação positiva e de fraca intensidade entre as reações centradas nos problemas e as reações que incentivam a expressão de emoções negativas $(r=0,35 ; p<0,01)$.
Por fim, destaca-se que a dimensão reações punitivas esteve negativamente correlacionada às reações que incentivam a expressão de emoções negativas $(r=-0,28$; $p<0,01)$. Além disso, as reações que ignoram as emoções negativas estiveram também negativamente correlacionadas às reações centradas no problema $(r=-0,34$; $p<0,01)$. A Tabela 2 apresenta os detalhes dessas análises.

\section{Discussão}

Este estudo teve como objetivo apresentar os dados preliminares da adaptação da CCNES para o uso com mães brasileiras de crianças entre três e oito anos. Verificou-se que os valores de consistência interna das subescalas de práticas de socialização emocional (apoiadoras e não apoiadoras) foram satisfatórios e que, como esperado, grande parte das dimensões que compõem essas subescalas esteve acima de 0,70 . As exceções envolveram as reações centradas nas emoções e as reações de desconforto.

Com exceção das duas dimensões que não obtiveram valores de alfa satisfatórios, os resultados deste estudo são próximos aos encontrados por Fabes et al. (2002). Resultados semelhantes também foram verificados por Coutu et al. (2002), ao adaptarem a CCNES para a versão francesa, com amostra canadense de pais e mães de pré-escolares, e por Altan-Aytun et al. (2013), ao adaptarem a escala para a versão turca. Com relação às dimensões que não obtiveram valores satisfatórios, destaca-se a dimensão reações de desconforto, que

Tabela 1

Correlações de Pearson entre os itens que compõem a dimensão reações de desconforto e o escore geral da dimensão

\begin{tabular}{|c|c|c|c|c|c|c|c|c|}
\hline & Item 1 & Item 3 & Item 4 & Item 5 & Item 6 & Item 9 & Item 11 & Item 12 \\
\hline $\begin{array}{l}\text { Escore geral das } \\
\text { reações de desconforto }\end{array}$ & $0,34^{* *}$ & $0,54^{* *}$ & $0,52^{* *}$ & $0,35^{* *}$ & $0,34^{* *}$ & $0,22^{*}$ & $0,54^{* *}$ & $0,64^{* *}$ \\
\hline
\end{tabular}

Nota: ${ }^{*} p<0,05 ;{ }^{* *} p<0,01$.

Tabela 2

Correlações de Pearson entre as dimensões da Coping with Children's Negative Emotions Scale

\begin{tabular}{|c|c|c|c|c|c|c|c|}
\hline & 1 & 2 & 3 & 4 & 5 & 6 & 7 \\
\hline \multicolumn{8}{|l|}{ Reações que não apoiam as emoções } \\
\hline \multicolumn{8}{|l|}{ 1. Reações punitivas } \\
\hline 2. Reações que ignoram as emoções & $0,39^{* *}$ & & & & & & \\
\hline 3. Reações que minimizam as emoções & $0,58^{* *}$ & $0,25^{* *}$ & & & & & \\
\hline 4. Reações de desconforto & $0,57^{* *}$ & $0,23^{* *}$ & $0,25^{* *}$ & & & & \\
\hline \multicolumn{8}{|l|}{ Reações que apoiam as emoções } \\
\hline 5. Reações centradas no problema & $-0,12$ & $-0,34^{* *}$ & $-0,12$ & $-0,14$ & & & \\
\hline 6. Reações centradas nas emoções & 0,07 & $-0,01$ & 0,10 & $-0,09$ & $0,50^{* *}$ & & \\
\hline 7. Reações que incentivam & $-0,28^{* *}$ & $-0,10$ & $-0,16$ & $-0,19^{*}$ & $0,35^{* *}$ & 0,13 & \\
\hline
\end{tabular}

Nota: ${ }^{*} p<0,05 ;{ }^{* *} p<0,01$. 
apresentou, inicialmente, valor de alfa extremamente baixo $(0,16)$ quando comparada às outras, assim como em relação aos valores obtidos nessa dimensão com a amostra norte-americana (Fabes et al., 2002). Uma possível explicação para esse achado é a presença de itens negativos. Segundo Barnette (2000), apesar dos itens com pontuação reversa serem utilizados na construção de escalas para atrair mais a atenção dos respondentes, evitando a concordância direta com base na forma de construção da frase e não com base no conteúdo, muitos pesquisadores têm ressaltado que o uso de itens com palavras ou sentido negativos prejudica a análise de algumas propriedades psicométricas, como a consistência interna. Ainda de acordo com Barnette, tem sido levantada a hipótese de que isso ocorreria porque os itens negativos não apresentam sentido exatamente oposto à escrita direta do item, o que dificultaria o entendimento a respeito do que o item avalia. Assim, após a constatação do baixo valor de alfa, os quatro itens negativos foram retirados para uma nova avaliação da consistência interna. Foi verificado aumento do alfa $(0,46)$, o que não representou, contudo, mudança expressiva, já que o valor permaneceu insatisfatório (Field, 2005). Como sugerido por Tavakov e Dennick (2011), foi realizada ainda uma análise de correlação entre os índices dessa dimensão e o escore geral da dimensão para verificar se alguma correlação teria valor igual a zero. Todas as correlações, no entanto, foram significativas e positivas, o que não sinaliza a necessidade de exclusão de nenhum dos itens. Outra sugestão apresentada por Tavakov e Denninck (2011) para explicar os baixos valores de alfa é o número de itens da dimensão. Segundo os autores, é possível que, após a retirada dos itens negativos, a dimensão tenha ficado com número insuficiente de itens para a avaliação da consistência interna. Contudo, para que essas hipóteses sejam avaliadas, é necessário ainda que os itens que compõem essa dimensão sejam mais bem investigados por meio de análise fatorial.

Fabes et al. (2002) verificaram índice de consistência interna satisfatório para as reações de desconforto. Entretanto, achados de outros estudos que envolveram a adaptação dessa escala para outras línguas e contextos - ou a aplicação da escala original em outra cultura - destacam a necessidade de revisão. $\mathrm{Na}$ adaptação da versão francesa, os valores de alfa da dimensão foram de 0,50 para a participação das mães e de 0,59 para a participação dos pais (Coutu et al., 2002). O estudo de Melo (2005), que buscou adaptar uma versão reduzida da CCNES, com oito situações, para aplicação com pais e mães portugueses, obteve valor de alfa de 0,42 , o qual, após a retirada dos itens negativos da dimensão, passou para 0,60. Altan-Aytun et al. (2013), ao avaliarem as práticas de socialização emocional adotadas por mães turcas de 141 crianças entre três e cinco anos, também verificaram a baixa qualidade da dimensão. Os autores levantaram a hipótese de que esses itens parecem envolver reações mais severas e punitivas que não destacam o desconforto da mãe, como, por exemplo, "digo a ele para não nos envergonhar com o seu choro".

De qualquer forma, é necessário que futuros estudos avaliem essa dimensão com mais cautela, especialmente quando aplicada a grupos distintos daquele para o qual a escala foi produzida. É possível, também, que as reações destacadas nos itens sejam mais características do grupo de pais norte-americanos ou que a escrita dos itens não esteja realmente adaptada para o contexto sociocultural ao qual a versão se destina, o que dificultaria sua compreensão por participantes de outros contextos. Caso isso esteja ocorrendo, seria recomendável, por exemplo, investir em avaliações preliminares realizadas por meio de estudos piloto, de forma que os grupos de pais possam oferecer alternativas para que os itens estejam mais próximos de sua linguagem e seu cotidiano, sem alterar a característica central do item, o que representa um desafio para os estudos que objetivam adaptar instrumentos de avaliação (Borsa et al., 2012).

Ao contrário da hipótese do presente estudo, que previa que as dimensões que compõem a subescala de reações que apoiam a expressão emocional de crianças, e dos achados do estudo de Fabes et al. (2002), nesta pesquisa não foram verificadas correlações significativas entre todas as dimensões que avaliam as reações que apoiam a expressão de emoções negativas por parte dos filhos. Foram verificadas duas correlações positivas significativas, uma entre as reações centradas no problema e as reações centradas nas emoções e outra entre as reações centradas no problema e as reações que incentivam. Por outro lado, as quatro dimensões que em conjunto avaliam as reações que não apoiam a expressão de emoções negativas correlacionaram-se de forma positiva entre si, de modo similar aos achados de Fabes et al. (2002) Além disso, as reações punitivas estiveram negativamente correlacionadas às reações que incentivam, enquanto as reações que ignoram estiveram negativamente correlacionadas às reações centradas no problema. Esses achados também são semelhantes aos reportados por Fabes et al. (2002) Entretanto, destaca-se que esses são resultados preliminares e que uma avaliação futura, com uma amostra maior, poderá oferecer dados mais consistentes a respeito das relações entre as dimensões e as subescalas e da distribuição em fatores. De qualquer modo, as correlações positivas indicam aproximações entre as dimensões que pertencem a uma mesma subescala (apoiadora ou não apoiadora), e as correlações negativas entre dimensões de diferentes subescalas sugerem a distinção entre ambas. Nesse sentido, os resultados satisfatórios das análises preliminares do processo de adaptação da escala indicam que é possível dar seguimento ao estudo, com o processo de validação da escala.

É válido ressaltar que no presente estudo foi incluída a dimensão que avalia as reações que ignoram as emoções 
negativas da criança, a qual não compõe a versão original da CCNES. Até o momento, provavelmente devido à sua inclusão recente, poucos estudos avaliaram as propriedades psicométricas dessas reações, o que limita a comparação dos achados deste estudo. Entretanto, Mirabile (2015) verificou correlações positivas entre essa dimensão e as demais dimensões da subescala de práticas não apoiadoras com exceção das reações de desconforto —, além de correlação negativa de fraca intensidade entre essa dimensão e as reações de incentivo. No presente estudo, também foram verificadas correlações positivas com as outras dimensões da subescala de práticas não apoiadoras, porém de fraca intensidade, além de relação negativa entre essa dimensão e as reações centradas no problema. Ainda assim, sua inclusão parece importante para tornar o instrumento mais completo, abrangendo maior diversidade de possibilidades de reações parentais frente às emoções dos filhos.

Em síntese, os achados da avaliação preliminar da versão brasileira da CCNES parecem indicar o bom funcionamento da escala, assim como a boa qualidade da adaptação linguística e cultural realizada. Ainda que o estudo não apresente grande número de participantes, necessário para análises mais refinadas, verificaram-se bons indicadores de consistência interna, além de importantes correlações entre as dimensões, resultados semelhantes aos achados do estudo que avaliou a versão original da escala (Fabes et al., 2002) e de outros estudos que avaliaram versões adaptadas do mesmo instrumento (Altan-Aytun et al., 2013; Coutu et al., 2002). Ainda assim, análises adicionais são necessárias, especialmente sobre a dimensão que avalia as reações de desconforto dos pais frente às emoções negativas dos filhos e a dimensão das reações que ignoram as emoções negativas dos filhos, devido à sua inclusão recente na escala. É válido ressaltar, também, que a escala foi aplicada apenas a mães de dois grandes centros urbanos, Rio de Janeiro e Salvador, o que pode constituir uma limitação importante a ser considerada em futuros estudos. Entretanto, destaca-se que este estudo envolveu a adaptação de um instrumento que avalia as práticas de socialização emocional, área pouco explorada no contexto brasileiro. Isso é particularmente importante, visto que boa parte das pesquisas já conduzidas sobre práticas de socialização emocional foi realizada com famílias brancas, de classe média e provenientes de culturas ocidentais, especialmente do hemisfério Norte, o que dificulta ou impossibilita a generalização dos achados para outros contextos socioeconômicos e culturais (Chaplin, Casey, Sinha, \& Mayes, 2010). Dessa forma, estudos que envolvam a construção ou a adaptação de instrumentos para diferentes contextos podem contribuir para a ampliação do conhecimento a respeito das interações entre pais e filhos e de como os pais ensinam os filhos a descrever, lidar e expressar emoções consideradas difíceis, como tristeza, raiva e medo, desde os primeiros anos de vida.

\section{Referências}

Altan-Aytun, Ö., Yagmurlu, B., \& Yavuz, H. M. (2013). Turkish mothers' Coping with Children's Negative Emotions: a brief report. Journal of Child and Family Studies, 22(3), 437-443. doi: 10.1007/s10826-012-9597-x

Alves, D., \& Cruz, O. (2011). Reações parentais às emoções negativas dos filhos (RPEN): um questionário da avaliação da meta-emoção parental. In A. S. Ferreira, A. Verhaeghe, D. R. Silva, L. S. Almeida, R. Lima, \& S. Fraga (Orgs.), Atas do VIII Congresso Iberoamericano de Avaliação/Evaluación Psicológica e XV Conferência Internacional Avaliação Psicológica: Formas e Contextos (p. 1480-1492). Lisboa: SBP. Recuperado de http://hdl.handle.net/10216/57244

Barnette, J. J. (2000). Effects of stem and Likert response option reversals on survey internal consistency: if you feel the need, there is a better alternative to using those negatively worded stems. Educational and Psychological Measurement, 60(3), 361-370. Recuperado de http://epm.sagepub.com/content/60/3/361.full.pdf+html

Borsa, J. C., Damásio, B. F., \& Bandeira, D. R. (2012). Adaptação e validação de instrumentos psicológicos entre culturas: algumas considerações. Paidéia, 22(53), 423-432. doi: 10.1590/1982-43272253201314

Cassano, M., Zeman, J., \& Sanders, M. R. (2014). Responses to children's sadness: mothers' and fathers' unique contributions and perceptions. Merrill-Palmer Quarterly, 60(1), 1-23. doi: 10.13110/merrpalmquar1982.60.1.0001

Chaplin, T. M., Casey, J., Sinha, R., \& Mayes, L. C. (2010). Gender differences in caregiver emotion socialization of low-income toddlers. In A. K. Root \& S. A. Denham (Orgs.), New Directions for Child and Adolescent Development, 128, (p. 11-27). São Francisco: Jossey-Bass. doi: $10.1002 / \mathrm{cd}$

Coutu, S., Dubeau, D., Provost, M. A., Royer, N., \& Lavigueur, S. (2002). Validation de la version française du questionnaire Coping with Children's Negative Emotions Scale-CCNES. Revue Canadienne des Sciences du Comportement, 34(4), 230-234. doi: 10.1037/h0087175

Eisenberg, N., Cumberland, A., \& Spinrad, T. L. (1998). Parental socialization of emotion. Psychological Inquiry, 9(4), 241-273. Recuperado de http://www.ncbi.nlm.nih.gov/pmc/articles/PMC1513625/pdf/nihms-10706.pdf

Fabes, R. A., Eisenberg, N., \& Bernzweig, J. (1990). The Coping with Children's Negative Emtion Scale: procedures and scoring. Disponibilizado pelos autores. Arizona State University.

Fabes, R. A., Poulin, R. E., Eisenberg, N., \& Madden-Derdich, D. A. (2002). The Coping with Children's Negative Emotions Scale (CCNES): psychometric properties and relations with children's emotional competence. Marriage E Famiy Review, 34(3-4), 285-310. doi: 10.1300/J002v34n03_05

Field, A. (2005). Discovering statistics using SPSS. Londres, GB: Sage Publications.

Halberstadt, A. G., Parke, R. D., Cassidy, J., Stifter, C. A., \& Foz, N. A. (1995). Self-expressiveness within the family context: psychometric support for a new measure. Psychological Assessment, 7(1), 93-103. doi: 10.1037/1040-3590.7.1.93 
Havighurst, S. S., Wilson, K. R., Harley, A. E., \& Prior, M. R. (2009). Tuning in to kids: an emotion-focused parenting program — initial findings from a community trial. Journal of Community Psychology, 37(8), 1008-1023. doi: 10.1002/jcop

Lagacé-Séguin, D. G., \& Coplan, R. J. (2005). Maternal emotional styles and child social adjustment: assessment, correlates, outcomes and goodness of fit in early childhood. Social Development, 14(4), 613-636. doi: 10.1111/j.1467-9507.2005.00320.x

Manzi-Oliveira, A. B., Balarini, F. B., Marques, L. A. S., \& Pasian, S. R. (2011). Adaptação transcultural de instrumentos de avaliação psicológica: levantamento dos estudos realizados no Brasil de 2000 a 2010. Pisco-USF, 16(3), 367-381. doi: 10.1590/S1413-82712011000300013

Melo, A. I. M. T. (2005). Emoções no período escolar: estratégias parentais face à expressão emocional e sintomas de internalização e externalização da criança. (Dissertação de Mestrado). Universidade do Minho, Braga. Recuperado de https://repositorium.sdum.uminho.pt/ bitstream/1822/4926/1/tese mestrado ana melo.pdf

Mirabile, S. P. (2015). Ignoring children's emotions: a novel ignoring subscale for the Coping with Children's Negative Emotions Scale. European Journal of Developmental Psychology, 12(4), 459-471. doi: 10.1080/17405629.2015.1037735

Morris, A. S., Silk, J., Steinberg, L., Myers, S. S., \& Robinson, L. R. (2007). The role of the family context in the development of emotion regulation. Social development (Oxford, England), 16(2), 361-388. doi: 10.1111/j.1467-9507.2007.00389.x

Ramsden, R. S., \& Hubbard, J. A. (2002). Family expressiveness and parental emotion Coaching: their role in children's emotion regulation and aggression. Journal of Abnormal Child Psychology, 30(6), 657-667. doi: 0091-0627/02/1200-0657/0

Raval, V. V, \& Martini, T. S. (2011). "Making the child understand:" socialization of emotion in urban India. Journal of Family Psychology, 25(6), 847-856. doi: 10.1037/a0025240

Reis, A. H., Oliveira, S. E. S., Bandeira, D. R., \& Sperb, T. M. (2013). Adaptação e validação do Emotion Regulation Checlist (ERC). 43a Reunião Anual da Sociedade Brasileira de Psicologia. Aracaju-SE: Adaptação e validação do Emotion Regulation Checlist (ERC). Aracaju-SE.

Sroufe, L. A. (1996). Emotional development: the organization of emotional life in the early years. Nova Iorque: Cambridge University Press.

Tao, A., Zhou, Q., \& Wang, Y. (2010). Parental reactions to children's negative emotions: prospective relations to Chinese children's psychological adjustment. Journal of Family Psychologyology, 24(2), 135-144. doi: 10.1037/a0018974

Tavakol, M., \& Dennick, R. (2011). Making sense of Cronbach's alpha. International Journal of Medical Education, 2, 53-55. doi: 10.5116/ ijme.4dfb.8dfd

\section{Sobre as autoras}

Taiane Lins é graduada (2010). Mestre (2013) e doutoranda em Psicologia pela Universidade Federal da Bahia (UFBA). É professora na Faculdade Anísio Teixeira (FAT).

Patrícia Alvarenga é professora associada do Instituto de Psicologia da Universidade Federal da Bahia (UFBA). Mestre (1999) e doutora (2004) em Psicologia pela Universidade Federal do Rio Grande do Sul (UFRGS), com pós-doutorado (2013) pela Universitat de València (UV).

Deise Maria Leal Fernandes Mendes é professora adjunta na Universidade Estadual do Rio de Janeiro (UERJ). Mestre (2003) e Doutora (2008) em Psicologia pela Universidade Estadual do Rio de Janeiro (UERJ).

Luciana Fontes Pessôa é professora adjunta e coordenadora de Graduação do Departamento de Psicologia da Pontifícia Universidade Católica do Rio de Janeiro (PUC-Rio). Mestre (2004) e Doutora (2008) em Psicologia pela Universidade Estadual do Rio de Janeiro (UERJ). 